\title{
LES AGENTS DE LA DIPLOMATIE DES SOUVERAINS MAGHRÉBINS AVEC LE MONDE CHRÉTIEN (XII $-\mathrm{XV}^{\mathrm{e}}$ SIËCLE)
}

\author{
THE ACTORS OF THE MAGHRIBIAN DIPLOMACY \\ WITH THE CHRISTIAN WORLD \\ (12th-15th CENTURIES)
}

Résumé: Une des originalités de la pratique diplomatique des souverains maghrébins en direction des Etats chrétiens du nord réside dans l'envoi d'ambassadeurs de profils très variés. Alors que les ambassades envoyées à d'autres souverains du monde de l'Islam sont tous musulmans, éventuellement juifs, on trouve de nombreux chrétiens comme ambassadeurs chargés de missions auprès des puissances ibériques ou italiennes. Mercenaires ou marchands le plus souvent, ils ont certes une bonne connaissance des relations internationales de l'époque. Mais ils n'en ont pas moins un statut ambigu dans le cadre des relations islamo-chrétiennes en Méditerranée. Les souverains maghrébins utilisent en réalité, selon les besoins de la mission, des agents chrétiens et musulmans, mais tout en gardant un contrôle efficace sur les décisions prises.

Mots clés: Maghreb; Péninsule Ibérique; Italie; Relations internationales; Ambassades; Mercenaires; Marchands.
DOMINIQUE VALÉRIAN

Université Paris 1 Panthéon-Sorbonne UMR 8167 Orient et Méditerranée

Abstract: One of the originalities of the diplomatic practice of the Maghrebian sovereigns with northern Christian States resides in the use of very different types of ambassadors. Whereas the embassies sent to other sovereigns of the world of Islam are all Moslem, eventually Jewish, one finds many Christians as ambassadors sent for missions to Iberian or Italian States. As mercenaries or merchants mostly, they have certainly a good knowledge of the international relations of the time. But they have also a very ambiguous statute within the framework of the Islamo-Christian relations in the Mediterranean. The Maghrebian sovereigns actually use, according to needs for the mission, Christian or Moslem agents, still keeping an effective control on important political decisions.

Keywords: Maghrib; Iberian Peninsula; Italy; International relations; Embassies; Mercenaires

Les relations diplomatiques entre souverains maghrébins et États latins deviennent, à partir du XII ${ }^{\mathrm{e}}$ siècle, particulièrement suivies, que ce soit en période de guerre ou de trêve. Les échanges d'ambassades sont motivés soit par des considérations de stratégie politique internationale, en vue d'aboutir à des alliances et des traités de paix, soit par la nécessité de régler des 
problèmes plus mineurs - notamment de pirates et de captifs - mais qui empoisonnent les relations diplomatiques et plus généralement la vie des hommes qui circulent en Méditerranée. Pour la couronne d'Aragon, ces échanges ont été magistralement explorés par Charles-Emmanuel Dufourcq puis María Dolores López Pérez, grâce à la très abondante documentation de la chancellerie de la couronne d'Aragon et du royaume de Majorque. Les sources italiennes, quant à elles, sont moins précises pour les périodes les plus anciennes, mais ont néanmoins fourni la matière d'études utiles sur les relations entre l'Italie et le Maghreb, principalement oriental.

On pourrait revenir, ici ou là, sur certains détails de cette trame événementielle, mais ce n'est pas ce qui nous occupe ici. L'histoire diplomatique a connu ces dernières décennies des évolutions importantes, dans une perspective plus anthropologique, qui l'a orientée notamment vers l'analyse des milieux d'ambassadeurs ${ }^{1}$. Mais si ces études sur les ambassadeurs latins sont abondantes, les agents de la diplomatie des États musulmans n'ont jusqu'à présent guère retenu l'attention. C'est donc à ces acteurs des relations internationales que nous nous intéresserons, et plus précisément sur ceux qui agissent pour le compte des souverains maghrébins.

Le terme de «milieu» n'est certes pas très approprié ici, tant on a l'impression que les souverains maghrébins font appel, en fonction des circonstances, à des personnages d'origines et d'horizons très disparates. Mais ce qui surprend surtout, au premier abord, est l'usage fréquent de nonmusulmans pour accomplir des missions diplomatiques en terre chrétienne. C'est là une originalité par rapport à ce que l'on peut observer des ambassades échangées entre souverains musulmans, à l'intérieur du Dâr al-Islâm. Mais c'est aussi une singularité par rapport à la pratique des États chrétiens qui, s'ils ont recours à l'occasion aux services des juifs ${ }^{2}$, ne font en revanche pas appel aux musulmans.

Dans le cas des relations internes au monde de l'Islam en effet, on ne trouve guère que des musulmans, peut-être occasionnellement des juifs. Le plus souvent il s'agit d'oulémas, d'hommes de religion, qui ont aussi des fonctions plus politiques à la cour. Ainsi en 677/1278-9 le sultan de Tunis envoie en ambassade aux Mérinides le cadi de Bougie, Abû l-'Abbâs alGhumârî́ $\hat{i}^{3}$, et au début du XIV ${ }^{\mathrm{e}}$ siècle c'est un autre cadi de Bougie, al-

\footnotetext{
${ }^{1}$ Stéphane PÉQUIGNOT, Au nom du roi: pratique diplomatique et pouvoir durant le règne de Jacques II d'Aragon (1291-1327), thèse sous la dir. de Jean-Marie MoEGLIN, Universite Paris Val de Marne, 2004, Nicolas DROCOURT, Ambassadeurs étrangers à Constantinople: movens de contacts, d'échanges et de connaissances partielles du monde byzantin (VIIIe-XII siècles), in Espaces d'échanges en Méditerranée. Antiquité et Moyen Age (F. CLEMENT, J. TOLAN et J. WILGAUX, éds.), Rennes, 2006, pp. 107-134. Négocier au Moyen Age, éd. María Teresa FERRER MALLOL, Jean-Marie MOEGLIN, Stéphane PEQUIGNOT, Manuel SÁNCHEZ MARTínEZ, Barcelone, 2005.

${ }^{2}$ Charles-Emmanuel DufouRCQ, L'Espagne catalane et le Maghrib aux XII' et XIVe siècles, de la bataille de Las Navas de Tolosa (1212) à l'avènement du sultan mérinide Abou l'Hassan, (1331), Paris, 1966, p. 139.

${ }^{3}$ IBN KHALDÛN, Histoire des Berbères, trad. W. MAC GuCKIN DE Slane, Alger, 1852-1856, nouv. éd. revue de P. CASANOVA, Paris 1956, IV, p. 54.
} 
Ghubrînî, qui est chargé d'une mission auprès du maître de Tunis, mission dont l'échec fut suivi par la disgrâce d'al-Ghubrînît ${ }^{4}$. De même, en 869/1465, c'est encore son grand cadi, Muhammad b. al-'Uqbânî, que le sultan abdelwadide de Tlemcen envoie à Tunis ${ }^{5}$. Parfois les souverains ont recours aux services d'hommes de négoce, comme le sultan de Grenade qui, au milieu $\mathrm{du} \mathrm{XV}^{\mathrm{e}}$ siècle, envoie au Caire le marchand Muhammad al-Bunyûlî sur un navire de commerce chargé de présents ${ }^{6}$. On trouve également des poètes, dont on retient les vers prononcés devant le souverain, mais qui avaient sans doute des fonctions moins littéraires ${ }^{7}$. Pour certaines missions plus importantes cependant, on envoie de grands personnages de la cour, parfois des princes de la famille régnante ${ }^{8}$. Ainsi le ministre andalou Ibn al-Khatîb effectua-t-il plusieurs ambassades pour les Nasrides, notamment au Maroc ${ }^{9}$. De même, le Hafside al-Mustansir envoie au Maroc une délégation de cheikhs almohades avec à leur tête leur chef, Yahyâ b. Sâlih al-Hintâtî, un des plus hauts personnages de l'État ${ }^{10}$.

Concernant les ambassades chrétiennes en terre d'Islam, trois groupes particuliers ont été mis en lumière par Roser Salicru ${ }^{11}$ : premièrement les officiers royaux liés à la frontière ou aux mudejars, notamment du royaume de Valence. Mais il semble que cette première catégorie soit surtout active avec le sultanat de Grenade, et moins avec le Maghreb, même s'ils ne sont pas totalement étrangers à ce qui se passe de l'autre côté du Détroit de Gibraltar.

${ }^{4}$ Robert BRUnSChVIG, La Berbérie orientale sous les Hafsides, I, Paris, 1940, p. 113.

${ }^{5}$ 'ABD AL-BÂSIT, Deux récits de voyages inédits en Afrique du Nord au XV siècle, 'Abd al-Bâsit b.Halîl et Adorne, trad. Robert BRUNSCHVIG, Paris, 1936, rééd. 2001, trad. p. 71. L'année précédente al-'Uqbânî avait déjà apporté des cadeaux au sultan de Tunis : ÁL-ZARKÁsHî̀, Ta'rîkh al-dawlatayni al-muwahhidiyya wa-l-hafsiyya, éd. Husayn YA'QÛ́Bî, Tunis, 1998, p. 308, trad. Edmond FAGNAN, Chronique des Almohades et des Hafsides attribuée à Zerkechi, Revue de la société archéologique de Constantine, 1894, et à part, Constantine, 1895, p. 257.

${ }^{6} \mathrm{G} . \mathrm{S}$. Colin, Contribution à l'étude des relations diplomatiques entre les musulmans d'Occident et l'Egypte au XV' siècle, in Mélanges Maspéro, t. III, Orient islamique (= Mémoires publiés par les membres de l'Institut français d archéologie orientale du Caire, t. 68), 1935-1940, p. 200-201. La lettre est datée du 13 jumâdâ I, an 5 (=845/1441-2 ou 855/1451).

${ }^{7}$ En 1366 le Nasride Muhammad V envoie en ambassade le poète Ibn al-Hâjj al-Numayrî auprès d'Abû Hammû Mûsâ II pour demander des secours contre les chrétiens. Rachel ARIÉ, Les relations entre le royaume nasride de Grenade et le Maghreb de 1340 à 1391 , in Relaciones de la Península ibérica con el Magreb siglos XIII-XVI. Actas del coloquio (Madrid'17-18 dec. 1987), M. GARCÍA ARENAL et M.J. VIGUERA, éd., Madrid 1988, p. 28 . Cet al-Numayri devint par là suite le secrétaire et conseillier du sultan mérinide Abû́ 'Inân. Voir aussi les vers prononces par Ibn al-Khatîb à la cour mérinide, qui contribuèrent, dit-on, à̀ convaincre le souverain marocain (Ibid. p. 35). Dans l'Occident latin également, un ambassadeur se doit d'avoir un beau discours. Jean-Marie MOEGLIN, Introduction: heurs et malheurs de la négociation du Moyen Age à l'époque moderne, in Négocier au Moyen Age, op. cit., p. 24

${ }^{8}$ IBN KHALDÛN, Histoire des Berbères, op. cit., IV, p. 53 : un neveu du sultan mérinide est envoyé à Tunis, accompagné par un chef de tribu importante.

${ }^{9} \mathrm{E}$. MOLINA LÓPEZ, Ibn al-Jat'îb: vínculo vital, político y cultural entre al-Andalus y el Magreb, in Al-Andalus y el norte de Africa : relaciones e influencias (F. ROLDÁN, P. BENEITO ARIAS, éds.), Séville, 2004, p. 175 ; R. ARIÉ, Les relations, loc. cit., p. 25, 35.

${ }^{10}$ IBn KHALDÛN, Histoire des Berbères, op. cit., IV, p. 149 ; voir aussi p. 150-152, d'autres délégations de cheikhs almohades envoyés par Abû̀ 'Asî̀a.

${ }^{11}$ Roser SALICRÚ I LLUCH, Más allá de la mediación de la palabra: negociación con los infieles y mediación cultural en la Baja Edad Media, in Négocier au Moyen Age, op. cit., p. 428- 
En revanche on trouve de nombreux membres de l'entourage des princes chrétiens et du haut personnel de la cour comme ambassadeurs. La deuxième catégorie regroupe les marchands en affaires avec les pays musulmans, parfois y résidant, que l'on rencontre également pour les relations diplomatiques des républiques italiennes ${ }^{12}$. Damien Coulon fait le même constat pour les relations avec l'Orient musulman, qu'il s'agisse des puissances italiennes ou de la couronne d'Aragon ${ }^{13}$. Enfin la troisième catégorie dégagée par Roser Salicru est constituée par les mudejars. Cependant là encore leur rôle semble moins important pour les relations avec le Maghreb qu'avec le sultanat de Grenade, et Damien Coulon note leur absence dans les ambassades avec l'Orient musulman ${ }^{14}$. À ce dernier groupe peuvent être adjoints les juifs, présents dans les missions diplomatiques aussi bien dans le sultanat nasride qu'au Maghreb ou en Orient ${ }^{15}$.

Pour les ambassades envoyées par les souverains maghrébins en terre chrétienne, les solutions adoptées sont beaucoup plus variées. Si on trouve bien des musulmans, on y reviendra, ce qui frappe avant tout est l'usage fréquent de chrétiens pour porter les messages des sultans hors du Dâr alIslâm, voire pour négocier avec les puissances chrétiennes.

La catégorie qui revient le plus souvent est celle des miliciens chrétiens, du moins pour les relations avec les puissances ibériques ${ }^{16}$. Les exemples sont nombreux, et je n'en prendrai que deux. Le premier est Bernat Segui, qui intervient entre 1302 et 1309 dans les relations entre Jacques II d'Arragon et les Mérinides. Charles-Emmanuel Dufourcq émet l'hypothèse qu'il était peut-être un marchand majorquin au départ, sur la base d'un document de 1297 qui parle d'un Segui, en société avec un certain al-'Abbâs de Tlemcen ${ }^{17}$. Mais c'est surtout comme chevalier de la milice qu'il nous apparaît dans la correspondance diplomatique entre les deux royaumes. Il semble que dès 1297 il parte s'engager comme homme d'armes au Maghreb ${ }^{18}$.

\footnotetext{
${ }^{12}$ Voir également Ch.-E. DufourCQ, L'Espagne catalane, op. cit., p. 518.

${ }^{13}$ Damien Coulon, Négocier avec les sultans de Méditerranée orientale à la fin du Moyen Age. Un domaine privilégie pour les hommes d'affaires?, in Négocier au Moyen Age, op. cit., p. $511-518$.

${ }^{14}$ Ibid., p. 513-514.

${ }^{15}$ R. SALICRÚ I LLUCh, Más allá de la mediación de la palabra loc. cit, p. 433 ; D. Coulon, loc. cit., p. 513. Parmi eux il faut noter la fréquence des médecins, proches des souverains souvent, et dont ils ont la confiance. Ch.-E. DUFOURCQ, L'Espagne catalane, op. cit., p. 139.

${ }^{16} \mathrm{Au} \mathrm{XV}^{\mathrm{e}}$ siècle le sultan hafside 'Uthmân envoie également auprès de la cour d'Alphonse le Magnanime, à Naples, le mercenaire génois Biagio Cibo. Francesco CERONE Alfonse il Magnanimo ed Abu "Omar Othmân, Trattative e negoziati tra il Regno di Sicilia ed il Regno di Tunisi (1432-57), "Archivio storico per la Sicilia Orientale", 10 (1913), p. 49, 73-74.

${ }^{17}$ Ch.-E. DufourcQ, L'Espagne catalane, op. cit., p. 348, 354.

${ }^{18}$ Carmen BATLLE I GALlART, Noticias sobre la milicia cristiana en el Norte de Africa en la segunda mitad del siglo XIII, in Homenaje al profesor Juan Torres Fontes, Murcie, Vol. 1, 1987, p. 132. A cette date il emprunte de I'argent a Domenec, Salom, frère de Bonanat qui a été ambassadeur auprès du sultan mérinide en 1301, argent qu'il doit rembourser dans le sultanat de Tlemcen. En 1297 à Tlemcen il peut aussi bien s'être mis au service des Abdelwadides que des Mérinides, qui alors assiègent la ville.
} 
Dès 1301 il est cité comme caïd de la milice ${ }^{19}$, et il est envoyé l'année suivante par Abû Ya'qûb auprès de Jacques II, porteur d'une lettre et accompagné d'un certain Abû l-'Abbâs, servicial du sultan mérinide ${ }^{20}$. Après être revenu au Maroc, il repart en 1303, accompagné par un Muhammad alJayr, qualifié de drogman. Au cours de cette mission il recrute des mercenaires pour la milice du sultan, alors que son frère Arnau est envoyé par le roi Jacques II au Maroc. Arnau rentre l'année suivante, avec une demande d'aide navale de la part du sultan, et il est là encore accompagné par un musulman, Abû 1-'Abbâs al-Tortosi. Impatient de ne pas recevoir de réponse à cette demande urgente, Abû Ya'qûb envoie une deuxième ambassade, menée par un autre chevalier de sa milice chrétienne, Bernat de Claramunt. Arnau Segui rentre finalement, avec pour mission de négocier les conditions de cette aide militaire $^{21}$. On retrouve Bernat Segui en 1308, lorsque Jacques II, devant le risque d'un rapprochement entre Mérinides et Nasrides, demande au nouveau sultan marocain Abû Thâbit de lui envoyer le caïd de sa milice, qui est resté bien en cour malgré la mort d'Abû Ya'qûb. Bernat part alors, accompagné d'Abû l-'Abbâs Ahmad ${ }^{22}$. Par la suite il ne semble pas qu'il ait été de nouveau envoyé en péninsule Ibérique, mais plusieurs de ses proches se voient confier des missions par les Mérinides: à la fin de l'année 1308 Pere Marti d'Orta, un de ses collaborateurs, est porteur d'une lettre pour Jacques II, à la suite de quoi un parent de Segui, Ramon Toro, accompagne un ambassadeur aragonais pour une négociation de paix ${ }^{23}$. Par ailleurs Bernat Segui reste en correspondance épistolaire avec la cour d'Aragon ${ }^{24}$. Il est en particulier le destinataire d'une des lettres envoyées par Jacques II à plusieurs personnes influentes de la cour mérinide en $1309^{25}$. Son rôle auprès du sultan apparaît d'ailleurs à travers deux lettres que lui adresse en 1302 et 1303 le Conseil des Cent de Barcelone pour qu'il facilite l'achat de blé au Maroc et intervienne dans le rachat de captifs ${ }^{26}$. On a donc affaire à un alcayt bien implanté dans les milieux dirigeants marocains, qui conserve son influence malgré les changements de sultans. Il dispose d'un réseau de relations, notamment

\footnotetext{
${ }^{19}$ María Dolores LóPEZ PÉREZ, La movilidad de la guerra : mercenarios catalanoaragoneses en las luchas maghrebies (ss. XIII-XIV), in La mobilité des personnes en Méditerranée de l'Antiquité à l'époque moderne : procédures de contrôle et documents d'identifications (Claudia MOATTI, éd.), Rome, $2004, \mathrm{p}_{\text {“ }} 416$ (citant Andres GIMENEZ SOLER, Caballeros españoles en Africa y africanos en España, "Revue Hispanique", 13 (1905), p. 305).

${ }^{20}$ Ch.-E. Dufourco, L'Espagne catalane, op. cit., p. 354-355; Id., Nouveaux documents sur la politique africaine de la Couronne d'Aragon, "Analecta Sacra Tarraconensia", 26 (1953), p. 301 .

${ }^{21}$ Ch.-E. DufourcQ, L'Espagne catalane, op. cit., p. 380.

${ }^{22}$ Ibid., p. 385.

${ }^{23}$ Ibid., p. 391-392.

${ }^{24}$ Il écrit à Jacques II une lettre portée par Ramon Toro et l'ambassadeur aragonais, et confie la même missive au secrétaire de la milice, Ramon Font, qui doit la porter par une autre voie, pour plus de sûreté. Ibid., p. 393.

${ }^{25}$ Parmi ces destinataires on relève le capitaine Sanchez de Troncones, le secrétaire de la milice Ramon Font, et un notable juif, Khalifat ben Roqqasa. Ibid., p. 393.

${ }^{26}$ Ibid., p. 358, 367.
} 
familial $^{27}$, aussi bien dans le sultanat que dans sa patrie d'origine, qu'il met à profit au cours de ces années. Par ailleurs, même si en 1308 Jacques II demande son intervention, c'est bien au nom du sultan qu'il agit. Enfin notons que dans ces négociations des années 1302-1308, l'aspect militaire est prédominant, le sultan du Maroc demandant une aide sous la forme soit d'une milice, soit de forces navales, dans le conflit qui l'oppose à Tlemcen et Ceuta.

Le second exemple de mercenaire employé comme ambassadeur est celui du bâtard Jacques d'Aragon, qui agit au service des sultans abdelwadides de Tlemcen, et plus particulièrement d'Abû Tashfîn, entre 1325 et 1329. Nous sommes là encore dans un contexte de conflit intra-maghrébin, les Abdelwadides cherchant à cette époque à s'emparer du port de Bougie et ayant pour cela besoin de l'aide de la flotte aragonaise. Fils de Jacques II d'Aragon et d'une Italienne, le bâtard Jacques est un des capitaines de la milice de Tlemcen, et à ce titre dirige une troupe, qui a son propre chapelain. Mais il n'est pas pour autant l'alcayt mayor des miliciens chrétiens, fonction détenue alors par un Majorquin, Guillem Estrus ${ }^{28}$. D'après Charles-Emmanuel Dufourcq, qui s'avance peut-être un peu trop sur ce point, il aurait acquis l'amitié de l'influent ministre Hilal, dit «le Catalan» en raison de ses origines, intime du sultan Abû Tashfîn qu'il a contribué à placer sur le trône en 1318. Il conduit sa première mission en 1325, à Valence, et en revient avec des propositions de paix de son père, qui demande l'envoi d'un plénipotentiaire pour signer le traité ${ }^{29}$. Deux ans plus tard le sultan l'envoie de nouveau, accompagné d'Ibn al-Haura. Jacques II mande alors le juge Lorenz Cima, qui exige, notamment, que le bâtard soit nommé chef de la milice de Tlemcen ${ }^{30}$. Le sultan joue alors la concurrence majorquine et envoie l'alcayt mayor, Guillem Estrus, auprès de la cour de Majorque. En 1329, et à nouveau en 1330, le bâtard Jacques est utilisé par le sultan pour discuter d'un traité, et pour la seconde ambassade il est accompagné encore de Ibn al-Haura et d'Abû Imrân Mûsâ b. Abî 'Inân ${ }^{31}$. Mais tous ces efforts ne permettent pas la signature d'un traité, et le bâtard Jacques finit par quitter le sultanat, en 1331 ou 1332, pour devenir un personnage important de la cour du roi d'Aragon ${ }^{32}$.

Ces alcayts interviennent donc régulièrement dans les négociations avec les puissances ibériques, principalement sur des questions d'alliances militaires, où le recrutement de miliciens par les sultans joue un rôle important.

\footnotetext{
${ }^{27} \mathrm{D}$ 'autres membres de sa fạmille sont ạlcayts dans le sultanat, à Marrakech et Rabat (Ibild., p. 462) et on a vu qu'il s'appuie à l'occasion sur certains de ses collaborateurs dans la milice, notamment son secrétaire. D'après Dufourcq, il est également proche de l'évêque du Maroc (Ibid., p. 388).

${ }^{28}$ Ibid., p. 472-473.

${ }^{29}$ Ibid., p. 483.

${ }^{30}$ Cima retourne en Aragon accompagné d'un nouvel ambassadeur abdelwadide, Abou-Corer far ben Abdallah. Ibid., p. 485.

${ }^{31}$ Ibid., p. 486.

${ }^{32}$ Ibid., p. 473 .
} 
Les marchands latins constituent la deuxième catégorie d'intermédiaires, et on trouve alors à la fois des sujets catalano-aragonais et des Italiens, très implantés dans le commerce maghrébin. Ainsi en 1278 le sultan de Tunis charge le Pisan Alberto Roncha d'une première mission auprès de Pierre III, afin d'obtenir des sauf-conduits pour les ambassadeurs qu'il compte envoyer à la cour d'Aragon ${ }^{33}$. De même, en 1336, les Hafsides envoient le marchand Francesc March, qui devient par la suite consul du roi d'Aragon à Collo et Bône ${ }^{34}$. $\mathrm{Au} \mathrm{XV}^{\mathrm{e}}$ siècle les Hafsides passent régulièrement par l'intermédiaire de marchands, génois le plus souvent, pour leurs relations avec la péninsule Ibérique ${ }^{35}$.

Ces quelques exemples appellent plusieurs remarques. On note tout d'abord que les marchands sont plus présents dans le domaine hafside ${ }^{36}$ qu'à Tlemcen ou chez les Mérinides, ce qui peut s'expliquer en partie par l'importance moindre des questions liées à la milice dans les relations diplomatiques entre l'Ifrîqiya et la couronne d'Aragon, et à l'inverse par la place plus grande des questions de commerce ou de rachats de captifs, qui concernent plus directement le milieu marchand ${ }^{37}$. Mais cela peut être également lié au fait que les Italiens, nombreux parmi ces ambassadeurs marchands, sont plus solidement implantés dans ces régions que dans les sultanats abdelwadide et mérinide. On note par ailleurs le nombre relativement important d'Italiens chargés de missions en terre catalane, ce qui peut certes s'expliquer par une meilleure implantation dans certaines régions, mais aussi peut-être par le souci d'avoir recours à des personnes jugées plus neutres et moins liées aux intérêts de la puissance catalane. Enfin, comme on l'avait déjà constaté pour les mercenaires, ces marchands sont souvent accompagnés dans leur mission par un sujet des sultans, juif ou musulman.

Ce rôle des marchands latins s'explique d'abord par leur facilité à se déplacer entre le Maghreb et l'Europe, mais aussi par les liens qu'ils peuvent entretenir avec les élites politiques de leur pays ou d'autres pays chrétiens. Certains appartiennent d'ailleurs à cette élite municipale, et peuvent y occuper

${ }^{33} I$ Ibid., p. 241. Le roi accorde alors garantie et protection aux ambassadeurs hafsides et à leur suite, aux bateaux qu'ils emprunteront et aux marchandises qu'ils transporteront.

${ }^{34}$ Robert BRUNSCHVIG, Documents inédits sur les relations entre la couronne d'Aragon et la Berbérie orientale au XIV" siècle, "Annales de l'Institut d'Etudes Orientales d'Alger", 2 (1936), p. 237-238. Il est alors accompagné par un musulman, "Alî b. Muhammad al-Ansârî, de la «cour" du sultan, et qui est peut-être un parent du négociateur de la paix de 1323, Zayt Ábinmahomet Alenzari. Voir aussi María Dolores LóPEZ PEREZ, La Corona de Aragón y el Magreb en el siglo XIV (1331-1410), Barcelone, 1995, p. 139.

${ }^{35} \mathrm{R}$. BRunSCHVIG, La Berbérie orientale, op . cit., p. 229, 232 ; R. SALICRÚ I LluCH, Más allá de la mediación de la palabra, loc. cit., p. 437.

${ }^{36} \mathrm{On}$ trouve également de mercenaires, comme les Cibo, à partir du XIV e siècle - mais ils sont issus d'une famille qui s'est illustrée dans le négoce au siècle précédent. Cf. Georges JEHEL, Les Cibo de Gênes, un réseau méditerranéen au Moyen Age, in Migrations et diasporas méditerranéennes ( $X^{e}-X V I^{e}$ siècles), M. BALARD et A. DUCELlER éds., Paris, 2002, pp. 285-296.

${ }^{37}$ En 1416 le Hafside Abû Fâris envoie ainsi le marchand vénitien Da Canale auprès dẹ la Sérénissime, porteur d'un message disant que le sultan est prêt à libérer les 80 captifs qui se disent vénitiens à condition d'avoir confirmation de leur "nationalité". Alberto SACERDOTI, Venezia e il regno hafsida di Tunisi : trattati e relazioni diplomatiche (1231-1534), "Studi veneziani", 8 (1966), p. 331. 
des postes importants, comme le montre le cas de Francesc March, qui devient consul après son ambassade. Ils sont par ailleurs de bons connaisseurs des réalités diplomatiques de leur temps, et notamment des enjeux commerciaux de part et d'autres, mais aussi des problèmes qui peuvent se poser dans le quotidien des relations entre l'Europe et le Maghreb. Enfin certains d'entre eux entretiennent des relations étroites et de confiance avec les souverains maghrébins. Ils sont à l'occasion leurs partenaires commerciaux, ou du moins leurs clients et fournisseurs. Mais certains poussent ces relations plus avant, et ont un accès privilégié à la cour et à l'entourage du souverain. C'est le cas par exemple du Catalan Rafael Vives, qui est installé depuis longtemps à Tunis, avant d'obtenir la ferme du corail de la côte nord en 1446, et que Philippe Gourdin qualifie de «Latin de cour ${ }^{38}$. On le voit en 1455 représenter le sultan de Tunis auprès du roi d'Aragon, puis en 1457 accompagner une ambassade hafside à Palerme, chargée par le sultan 'Uthmân de recenser les esclaves musulmans insolvables dans l'île $\mathrm{e}^{39}$. Cette proximité entre certains marchands latins et les cours maghrébines apparaît clairement dans un mémoire que remet Mateu Besanta (ou Besanca) à Pierre d'Aragon en 1353 relatif à une ambassade en partance: il rappelle qu'il avait déjà mené une mission dix ans plus tôt, et qu'un notable (mayor) de la cour lui avait alors transmis le message suivant du sultan: «Mateu, ton père était notre ami et "de casa nostra" et a négocié la paix avec le roi de Majorque; si tu traites la paix entre le roi d'Aragon et nous, nous te ferons beaucoup de bien ${ }^{40}$.

À ces Latins s'ajoutent souvent des juifs, qui peuvent être sujets des rois chrétiens comme des sultans musulmans. Les juifs ont toujours eu un rôle important dans les négociations diplomatiques, et sont employées des deux côtés ${ }^{41}$. Ainsi en 1294 , puis en 1295, le sultan mérinide envoie le juif Isaac, accompagné chaque fois par le marchand majorquin Francesc del Clergue. Mais il finit par rentrer demander l'envoi d'un plénipotentiaire habilité à négocier avec le roi d'Aragon ${ }^{42}$. De même, en 1400 c'est un médecin juif, Bondavi, qui est envoyé par le sultan Hafside auprès de Martin d'Aragon ${ }^{43}$. Comme les marchands, les juifs ont l'avantage de disposer de réseaux,

\footnotetext{
${ }^{38}$ Philippe GOURDIN Les relations politiques et économiques entre l'Italie tyrrhénienne et le Maghreb au XV siècle, Thèse sur travaux, Volume de synthèse, Paris, décembre 2001, p. 100.

${ }^{39} \mathrm{Ph}$. Gourdin Les relations politiques, op. cit., p. 118 ; R. SALICRÚ I LLUCH, Más allá de la mediación de la palabra, loc. cit., p. 437.

${ }^{40}$ Archives de la Couronne d'Aragon, Rg 555, f. 118 v-120 r (avant le 1/8/1353), éd. R. BRUNSCHVIG, Documents inédits, loc. cit., nº 16, p. 262.

${ }^{41}$ Pour les souverains chrétiens, cf. Ch.-E. DufourCQ, L'Espagne catalane, op. cit., p. 139. C'est là une tradition ancienne: déjà le calife abbasside de Bagdad avait envoyé un juif, Isaac, auprès de Charlemagne. Cf. Philippe SÉNAC, Le Monde carolingien et l'Islam, Paris, 2006, p. 47.

${ }^{42}$ Ch.-E. DufourcQ, L'Espagne catalane, op. cit., p. 231, 346.

${ }^{43} \mathrm{Le}$ roi le dispense, ainsi que ses deux coreligionnaires qui l'accompagnent, du port de la rouelle et autres marques distinctives. Peut-être ce Bondavi est-il le descendant d'un autre médecin juif du même nom, employé comme ambassadeur du roi d'Aragon en 1293 auprès du sultan de Tlemcen. R. BRUNSCHVIG, La Berberie, op. cit., p. 224, 413, à corriger avec CharlesEmmanuel DUFOURCO, La couronne d'Aragon et les Hafsides au XIII' siècle, "Analecta Sacra Tarraconensia”, 25 (1952), p. 89.
} 
notamment familiaux et commerciaux, qui s'étendent à la fois sur le Maghreb et l'Europe, et qui facilitent leurs déplacements et leur connaissance de la situation internationale ${ }^{44}$. Ils peuvent avoir par ailleurs, dans certains cas, des positions importantes à la cour. C'est le cas sans doute des médecins, dont on sait qu'ils appartenaient parfois à l'entourage proche des sultans, qui leur accordaient leur confiance ${ }^{45}$. Cette position sociale et politique nous est confirmée par une lettre envoyée en 1309 par Jacques II à un notable juif proche de la cour mérinide, Khalifa ben Roqqasa, pour lui recommander ses ambassadeurs ${ }^{46}$. Enfin ils ont des compétences linguistiques qui facilitent les discussions, et on les voit parfois intervenir comme traducteurs, par exemple pour les traités ${ }^{47}$.

Les drogmans apparaissent d'ailleurs souvent comme intermédiaires diplomatiques, là encore en raison à la fois de leurs compétences linguistiques mais aussi de leurs relations multiples et de leur connaissance des relations internationales et de leur complexité. Parfois ils accompagnent des ambassadeurs, et n'ont alors qu'un rôle technique ${ }^{48}$. Mais leur fonction peut être aussi plus politique, comme dans le cas de Saïd, interprète bougiote, envoyé en ambassade en Aragon en $1310^{49}$, ou encore le cas plus connu d'Anselme Turmeda, renégat hafside qui intervient dans des négociations à la fin du $\mathrm{XIV}^{\mathrm{e}}$ siècle $^{50}$.

Ce rôle important de chrétiens ou de juifs ne doit cependant mener à penser que les ambassadeurs musulmans étaient totalement absents. On trouve tout d'abord souvent des musulmans qui accompagnent les mercenaires ou les marchands. Ainsi Bernat et Arnau Segui, déjà évoqués plus haut, sont toujours flanqués d'un musulman: en 1302 c'est un certain Abû l-'Abbâs b. Ahmad ${ }^{51}$, puis en 1303 Muhammad al-Jayr, qui est qualifié de drogman ${ }^{52}$. On voit mal quelle peut être l'utilité d'un traducteur pour un Catalan qui mène une mission

${ }^{44} \mathrm{Cf}$. Dominique VALÉRIAN, Bougie, port maghrébin, 1067-1510, Rome, 2006, p. 525-534.

${ }^{45} \mathrm{Ce}$ rôle des médecins comme hommes de confiance des souverains se retrouve également pour les médecins musulmans, comme dans le cas d'Abû l-'Abbấs b. 'Ayshûn, ambassadeur d'Ibn al-Lihyânî en Aragon en 13311. Ch.-E. DUFOURCQ, L'Espagne catalane, op. cit., p. 500 ; R. BRUNSCHVIG, La Berberie, op. cit., p. 136.

${ }^{46} I b i d .$, p. 393. D'autres lettres sont envoyées à des personnages importants de la cour, comme les vizirs Rassoul et Ibrahim ben Isa, le caìd Ali, le conseiller du sultan Abdallah ben Modien, les capitaines Bernat Segui et Sanchez de Troncones, le secrétaire de la milice Ramon Font.

${ }^{47}$ R. BRUNSChVIG, La Berberie, op. cit., p. 413-414.

${ }^{48} \mathrm{C}$ 'est sans doute le cas par exemple en 1323 avec l'interprète Abû 'Uthmân Sa'îd, qui accompagne l'ambassadeur bougiote Abû Muhammad 'Abd al-Malik b. Abû l-Hasan 'Alî à la cour d'Aragon. Ch.-E. DUFOURCQ, L'Espagne catalane, op. cit., p. 505.

${ }^{49}$ Ch.-E. DufourCQ, L'Espagne catalane, op. cit., p. 499.

${ }^{50} \mathrm{R}$. BRunschvig, La Berberie, op. cit., p. 201. Voir également le cas du drogman Ibn Taghrî Birdî, ambassadeur mamelouk a Venise au début du XVI ${ }^{\mathrm{e}}$ siècle, qui était sans doute également un renégat. John WANSBROUGH, A Mamluk Ambassador to Venice in 913/1507, "Bulletin of the School of Oriental and African Studies", 26/3 (1963), pp. 503-530.

${ }^{51}$ Ch.-E. DufOURCQ, L'Espagne catalane, op cit., p. 354-355; ID., Nouveaux documents, loc. cit., p. 302. Il est qualifié de «servicial» du sultan mérinide.

${ }^{52}$ Ch.-E. DufourCQ, L'Espagne catalane, op. cit., p. 363. 
en Catalogne, et il faut donc penser que cet al-Jayr a là une fonction politique, et non de simple traduction. L'année suivante Arnau est accompagné d'Abû 1-'Abbâs al-Tortosi, qui est peut-être le même que le Abû l-'Abbâs de 1302, que l'on retrouve d'ailleurs en 1308, toujours aux côtés de Bernat Segui ${ }^{53}$. On aurait alors là un personnage de l'entourage du sultan mérinide Abû Ya'qûb spécialisé dans les relations diplomatiques avec l'Aragon. Sa nisba, al-Tortosi, montre qu'il a des origines andalouses, sans que l'on puisse affirmer avec certitude qu'il a lui-même vécu en péninsule Ibérique. Mais ce lien, même ancien, a pu jouer un rôle dans sa connaissance des réalités du monde chrétien.

On rencontre également des ambassades musulmanes venant en Europe sans être accompagnées par des Latins. Il n'est cependant pas toujours facile d'identifier ces personnages, dont l'orthographe est souvent déformée par la graphie latine ${ }^{54}$, et que l'on ne retrouve pas forcément dans les sources arabes contemporaines. Parfois cependant on peut repérer quelques personnalités de haut rang, comme le vizir mérinide 'Abd al-Haqq, envoyé à plusieurs reprises en Castille à la fin du XIIIe siècle ${ }^{55}$, ou le vizir Tlemcénien Ibn Beridi qui mène plusieurs missions en Castille et en Aragon ${ }^{56}$. De même, en 1304 le sultan hafside envoie un cheikh nommé Abû Yahyâ b. Abû 1-'Abbâs, que Charles-Emmanuel Dufourcq identifie au puissant cheikh des Almohades et futur sultan Ibn al-Lihyânî, pour se plaindre de violations de la paix ${ }^{57}$. On le retrouve dix ans plus tard à Barcelone, pour une autre ambassade ${ }^{58}$. En 1315 c'est son propre frère que l'éphémère «roi de Miliana», cousin rebelle du sultan de Tlemcen, envoie en ambassade à Jacques II $^{59}$. Et en 1283 c'est un fils du sultan de Tunis qui est ambassadeur auprès du roi d'Aragon, sans que l'on sache cependant s'il est le fils du prince régnant ${ }^{60}$. Certaines de ces ambassades sont marquées par une pompe remarquable, comme celle menée en 1442 par un envoyé du sultan hafside 'Uthmân, Sîdî Ibrâhîm b. 'Alî,

${ }^{53}$ Ch.-E. DufourcQ, L'Espagne catalane, op. cit., p. 385-386.

${ }^{54}$ Les textes arabes sont souvent très discrets sur ces ambassades en pays infidèle, dont la légitimité n'est pas très sûre. Il faut donc le plus souvent s'appuyer sur la documentation latine, qui n'est pas toujours précise. Parfois on trouve simplement la mention d'une ambassade d'un souverain maghrébin, avec un simple prénom, souvent très courant et donc impossible à identifier. Dans certains cas les ambassades ne sont connues que par. les dépenses faites à cette occasion, et qui apparaissent dans les comptes royaux, sans même d'indication de nom.

${ }^{55}$ Ch.-E. DufourcQ, L'Espagne catalane, op. cit., p. 231.

${ }^{56}$ Ibid., p. 313-314.

${ }^{57}$ Ibid., p. 433.

${ }^{58} \mathrm{Il}$ en profite pour vendre du grain à Barcelone, et reçoit alors un sauf-conduit royal valable pour tous les voyages qu'il fera par la suite pour commercer. Charles-Emmanuel DUFOURCQ, Le commerce du Maghreb médiéval avec l'Europe chrétienne et marine musulmane : données connues et problemes en suspens, in Actes du congrès d'histoire et de civilisation du Maghreb, Tunis, 1974, Cahiers du CERES, Série histoire, 1, Tunis, 1979, p. 168.

${ }^{59}$ Charles-Emmanuel DufourCQ, Nouveaux documents sur la politique africaine de la Couronne d'Aragon, "Analecta Sacra Tarraconensia", 26 (1953), pp. 291-323, p. 292.

${ }^{60}$ Ch.-E. DufourcQ, La couronne d'Aragon et les Hafsides au XIII siècle, loc. cit, p. 61. 
qualifié de «noble seigneur Abramo» ${ }^{61}$, venu à Naples sur une galère battant pavillon de Tunis avec une nombreuse suite, interprètes, trompettes et tambours, et deux chevaux arabes magnifiques à offrir à Alphonse le Magnanime $^{62}$.

Comme dans le cas des ambassadeurs chrétiens ou juifs, on constate que certains envoyés musulmans sont également marchands, ou du moins pratiquent le commerce ${ }^{63}$. C'est le cas par exemple en $1250 \mathrm{du}$ messager du sultan abdelwadide Yaghmurasân, Abû Arlan, qui profite de sa mission pour faire des achats et des ventes en Catalogne, mais aussi à Montpellier, nouant des relations d'affaires avec un capitaliste de Barcelone, Ramon de Bagnares, qui s'intéresse depuis longtemps aux marchés ifrîqiyens ${ }^{64}$. On ignore s'il agit pour son propre compte ou au nom du sultan, mais on peut constater que les produits échangés - achats d'agrès, produit considéré comme stratégique, et vente de grandes quantités d'alun, produit qui peut faire parfois l'objet d'un monopole d'État- laissent supposer un lien étroit avec le pouvoir politique. De même en 1362 l'ambassadeur hafside Hamet ben Bien est qualifié de marchand dans la lettre de créance adressée à Pierre le Cérémonieux ${ }^{65}$. Enfin en 1440 Sidi Elyagi Mayamet ben Ysarb Adar, «ambassadeur du roi de Tunis», est à Trapani et achète au nom du sultan 744 salmas de froment qu'il fait expédier à Tripoli ${ }^{66}$.

Globalement cependant on constate que les ambassades envoyées par les États chrétiens en direction du Maghreb sont plus nombreuses que celles qui se font dans l'autre sens. Pour la seconde moitié du XIII ${ }^{\mathrm{e}}$ siècle, Dufourcq

\footnotetext{
${ }^{61}$ Il s'agit sans doute du même personnage qui apparaît dans les actes du notaire trapaniote Milo en mai 1443, sous le nom de Abram Bennoli et qualifié de «sarracenus accaydus et dominus assertus dohane civitatis thinisii et ad presens ambassiator Regis Thunisii». Carmello TRASSELLI, Sicilia, Levante e Tunisia nei sec. XIV e XV in Mediterraneo e Sicilia all'inizio dell'epoca moderna, Cosenza, 1977 [1 $1^{\text {re }}$ éd. Trapani, 1952], p. 330. On aurait alors l'intervention, comme ambassadeur, du chef de la douane de Tunis, un des premiers personnages de l'Etat, et le plus impliqué dans les relations avec les puissances latines.

${ }^{62}$ Francesco CERONE, Alfonse iI Magnanimo ed Abu 'Omar Othmân, Trattative e negoziati tra il Regno di Sicilia ed il Regno di Tunisi (1432-57), "Archivio storico per la Sicilia Orientale", $9(1912)$, p. 63.

${ }^{63}$ Il peut s'agir d'hommes de pouvoir qui font également des affaires. Cf. le cas du vizir tlemcennien qui effectue plusieurs missions diplomatiques en Castille et en Aragon, et qui en 1277 se voit remettre par Pierre III un sauf-conduit permanent (tant que dure la paix), pour qu'il puisse commercer librement et exporter des marchandises, en son nom et celui de son frère, du sultanat vers les pays de la couronne d'Aragon (Ch.-E. DUFOURCQ, L'Espagne catalane, op. cit., p. 313). Sur la place des hommes de pouvoir dans le grand commerce, cf. Dominique VALÉRIAN, Les élites politiques et l'activité économique des ports musulmans (XIII $-X V^{e}$ siècle), "Histoire urbaine", 19 (2007), pp. 117-128.

${ }^{64}$ Ch.-E. DufourCQ, L'Espagne catalane, op. cit., p. 145-146.

${ }^{65}$ Éd. Andrés GIMENEZ SOLER, Documentos de Tunez, originales o traducidos, "Anuari de l'Institut d'Estudis Catalans", 3 (1909-10), n' 34, p. 259 (10/6/1362). Il reçoit alors un saufconduit spécial accordé par le gouverneur de Majorque Bernat de Tous, pour transporter un chargement de blé depuis Tunis sur une nave majorquine et mener à bien plusieurs oppérations commerçiales. Il transporte notamment des cuirs de Tunis, de la soie nasride qu'il apporte d'Almeria. Il vient également à plusieurs reprises dans l'île avec des marchandises de Tunis: en avril 1363, avec Bernat Rivelar (Archivo del Reino de Mallorca, Real Patrimonio 3988/2, f. 34 v, $5 / 4 / 1363$ ); en juillet sur la nau de F. Serong (ibid. f. $37 \mathrm{v}, 15 / 7 / 1363$ ), et en septembre sur la nau de Huguet Valenti (ibid., f. $16 \mathrm{v}, 19 / 9 / 1363$ ). Sans doute à la même date il vend des marchandises venant de Minorque (ibid., f. 16 r) et 4 costals de soie d'Almeria (id).

${ }^{66}$ C. Trasselli, Sicilia, Levante et Tunisia, loc. cit, p. 129. Le document précise que cet ambassadeur «comprend notre langue».
} 
compte ainsi 4 ambassades hafsides en Aragon, pour 17 ambassades catalanes en Ifrîqiya ${ }^{67}$. Il semble qu'au $X^{\mathrm{e}}$ siècle le déséquilibre soit moins net ${ }^{68}$, mais il ne disparaît pas totalement. Et dans ces ambassades envoyées par les sultans en terre chrétienne, les musulmans semblent, globalement, moins nombreux que les chrétiens ${ }^{69}$. Sans doute y a-t-il en partie un effet de source, les documents latins rapportant davantage les ambassades envoyées par les Etats chrétiens. En outre, ces documents retiennent davantage les noms des ambassadeurs lorsque ceux-ci sont latins, alors que l'on a de nombreuses mentions de missions envoyées par les souverains musulmans, mais sans précision sur l'identité de l'ambassadeur ou du messager. Mais il n'en reste pas moins qu'il y a une originalité dans cette utilisation récurrente de chrétiens dans les missions mandatées par les sultans, qui ne manque pas de surprendre et pose certains problèmes.

Tout d'abord il faut expliquer ce recours à des chrétiens, alors même que pour les relations à l'intérieur du Dâr al-Islâm les ambassadeurs sont toujours des musulmans. On peut tout d'abord remarquer qu'à cette époque, d'une manière générale, les musulmans maghrébins ne se rendent guère en terre chrétienne, même si on en trouve à l'occasion, notamment à Valence ou Majorque $^{70}$. Le commerce, on le sait, est très largement pris en main par les Latins. Il a pu alors y avoir des réticences, côté maghrébin, à envoyer des ambassadeurs dans des pays considérés traditionnellement comme des terres de guerre. On connaît les réserves émises par les juristes au séjour et même au commerce des musulmans dans l'Europe chrétienne ${ }^{71}$. De même la signature de traités de paix avec des souverains infidèles, si elle n'est pas interdite, est strictement encadrée par le droit et continue de susciter parfois la désapprobation des oulémas ${ }^{72}$. Or ce sont ces hommes de religion qui constituent l'essentiel des ambassadeurs échangés traditionnellement avec les autres souverains musulmans. De fait, les personnes que l'on parvient à identifier dans les missions diplomatiques maghrébines appartiennent plutôt au monde du pouvoir ou du négoce, même si ces catégories sont loin d'être

\footnotetext{
${ }^{67}$ Ch.-E. DuFOURCQ, La couronne d'Aragon et les Hafsides au XIII siècle, loc. cit., pp. 108113. On rencontre le même déséquilibre pour les relations avec l'Orient musulman : sur 20 traités signés entre Venise et les Mamelouks, seul un a été négocié en Italie par des ambassadeurs du sultan. J. WANSBROUGH, A Mamluk Ambassador, loc. cit., p. 503.

${ }^{68}$ Cf. F. CERONe, loc. cit., passim.

${ }^{69}$ Là encore, il semble que ce déséquilibre soit moins évident au XV ${ }^{\mathrm{e}}$ siècle, notamment sous le sultanat de 'Uthmân en Ifrîqiya. Mais il faudrait une étude plus poussée pour s'en assurer.

${ }^{70}$ Dominique VALÉRIAN, Ifrîqiyan Muslim Merchants in the Mediterranean at the End of the Middle Ages, "Mediterranean Historical Review", 14/2 (1999), p. 47-66.

${ }^{71}$ Farhat DACHRAOUI, Intégration ou exclusion des minorités religieuses. La conception islamique traditionnelle in L'expulsió dels moriscos : consequències en el món islàmic $i$ el món cristià, Barcelone, 1994, p. 199-200.

${ }^{72}$ En 1270 c'est un chef de tribu qui négocie le traité avec les croisés, et un ouléma refuse de figurẹr comme témoin - et de fait ni le grand cadi, ni les deux chefs de la chancellerie ne figurent parmi les témoins. R. BRUNSCHVIG, La Berbérie, op. cit., p. 63 . Dans d'autres traités cependant les hommes de religion apparaissent bien parmi les témoins. Cf. Ibid., p. 66.
} 
étanches et fermées aux oulémas ${ }^{73}$. Mais il ne faut pas donner trop d'importance à ces difficultés juridiques, et les exemples relativement nombreux de musulmans se rendant en ambassade en terre chrétienne montrent que ces réticences pouvaient facilement être surmontées. Il faut donc chercher une autre explication à l'emploi des chrétiens par les souverains musulmans.

La rareté des déplacements, notamment marchands, de musulmans en terre chrétienne a en fait deux conséquences importantes, et plus pratiques. La première est que très naturellement les chrétiens se déplacent plus facilement en terre d'Islam que l'inverse. La seconde est que les musulmans ont une connaissance assez faible des réalités du monde européen. Les problèmes du voyage pouvaient cependant être assez aisément résolus: les musulmans pouvaient embarquer sur des navires chrétiens, ce qui était une pratique courante. Leur sécurité était en principe garantie par des sauf-conduits, qui les protégeaient contre les divers périls qui les menaçaient en terre chrétienne ou sur mer -à condition bien sûr que les ordres du souverain qui émettait ce sauf-conduit soient respectés. Ainsi en 1278 le sultan hafside décide d'envoyer des ambassadeurs à la cour d'Aragon, mais charge au préalable un Pisan, Alberto Roncha, de demander au roi un sauf-conduit pour ces ambassadeurs ${ }^{74}$. De même, en 1274, le roi d'Aragon Jacques le Conquérant, tout en autorisant la course contre les sujets du sultan de Tlemcen, interdit de s'en prendre à deux ambassadeurs musulmans envoyés par l'Abdelwadide auprès de la cour de Castille ${ }^{75}$. La procédure existe donc, même si elle reste un peu longue et surtout d'application incertaine.

La deuxième conséquence de cette faiblesse du commerce musulman dans l'Europe chrétienne est une méconnaissance certaine de ces pays. Les sultans n'ont pas non plus de consuls susceptibles de les informer sur la situation politique et économique, ni sur les codes en usage dans les cours européennes $^{76}$. Enfin les musulmans ont rarement la maîtrise des langues latines. Tout cela donne un avantage incontestable aux chrétiens, qu'ils soient marchands ou mercenaires, pour effectuer correctement les missions qui leurs sont confiées, et explique aussi le déséquilibre constaté dans les échanges d'ambassades entre celles envoyées par les souverains musulmans et celles envoyées par les chrétiens.

Mais cet emploi de chrétiens ne va pas sans poser des problèmes sur leur allégeance. Il est certain, comme le remarque Roser Salicru, qu'ils peuvent inspirer une plus grande confiance dans les cours européennes que des

${ }^{73}$ Il faut relever que dans l'autre sens les religieux sont assez rarement utilisés pour des missions diplomatiques en terre d'Islam. Le cas du frère bénédictin Giuliano Mayali, envoyé par Alphonse le Magnanime à Tunis reste relativement isolé. Cf. Francesco GIUNTA, Fra Giuliano Mayali, agente diplomatico di Alfonso il Magnanimo (1390 (?)-1470), "Archivio storico siciliano", sér. 3, vol. 2 (1947), p. 153-183.

${ }^{74}$ Ch.-E. DufourCQ, L'Espagne catalane, op. cit., p. 241.

${ }^{75}$ Ibid., p. 148.

${ }^{76}$ Sur l'importance de la maîtrise de ces codes, voir les remarques de R. SALICRÚ I LLUCH, Más allá de la mediación de la palabra, loc. cit., pp. 427, 431. 
ambassadeurs musulmans. Mais dans quelle mesure les sultans peuvent-ils s'appuyer, pour leur politique étrangère, sur des étrangers, infidèles qui plus est, et même le plus souvent sujets du souverain avec lequel ils correspondent? Ce problème se pose en particulier pour les caïds de la milice, dont on a vu le rôle important, notamment au Maroc et à Tlemcen. Ces caïds sont en général nommés par les rois chrétiens, et restent leurs sujets ${ }^{77}$. Indépendamment de la question religieuse, qui est souvent assez secondaire, il faut le reconnaitre, on peut se demander au service de qui agissent ces caïds. Pour Charles-Emmanuel Dufourcq, tout entier à sa réflexion sur l'impérialisme catalan, la chose ne fait guère de doute: ces caïds étaient un des instruments de la puissance des rois d'Aragon, et servaient leurs intérêts. Il l'affirme en particulier pour Bernat Segui ${ }^{78}$, ou le bâtard Jacques d'Aragon. À y regarder de plus près les choses sont plus compliquées. Tout d'abord il faut reconnaître que malgré leur rôle dans les négociations, les résultats pour le roi d'Aragon ne sont guère probants. Le bâtard Jacques semble plus préoccupé par le renforcement de sa propre position à la cour de Tlemcen, et notamment par sa rivalité avec l'alcayt mayor Guillem Estrus, que par le triomphe des positions de son père dans le sultanat. Quant à Segui, il sert semble-t-il loyalement le sultan mérinide, à la tête de la milice. Sans minimiser l'ambiguïté de leur position, il semble que ces caïds aient surtout une grande proximité avec les sultans qu'ils servent, d'autant que leur carrière dépend de leur fidélité absolue pour leur maître. On pourrait retrouver une semblable ambiguïté avec les marchands utilisés comme ambassadeurs par les sultans, qui ont aussi leurs stratégies propres, au profit de leur nation, de leur groupe socio-professionnel, voire de leurs propres affaires commerciales.

Ce qui apparaît pourtant c'est que les intérêts des sultans ne passent jamais au second plan, ce qui montre que les États chrétiens sont loin de manipuler à leur guise ces ambassadeurs. C'est même plutôt l'inverse qui se produit, les sultans instrumentalisant à l'occasion ces chrétiens en comptant sur la confiance qu'ils inspirent dans leur pays. C'est le cas par exemple avec le Hafside Ibn al-Lihyani, qui développe ce que l'on peut considérer comme une véritable stratégie de séduction, voire de désinformation, des puissances chrétiennes en faisant croire qu'il est prêt à apostasier. On le voit faire des confidences, sous le sceau du secret, à divers chrétiens proches de la cour, et notamment au chef des mercenaires Guillem de Moncada, qui font passer l'information auprès de Jacques II et d'autres souverains chrétiens ${ }^{79}$.

Il faut donc s'interroger sur la fonction précise de ces chrétiens envoyés par les sultans. Ils apparaissent en réalité davantage comme des

\footnotetext{
${ }^{77} \mathrm{Cf}$. Roser SALICRÚ I LLuCH, Mercenaires castillans au Maroc au début du XV siècle, Migrations et diasporas méditerranéennes ( $X^{e}-X V I^{e}$ siècles), M. BALARD, A. DUCELLIER, dir., Paris, 2002, p. 419-420. Robert Brunschvig note cependant une tendance, au XV ${ }^{\mathrm{e}}$ siècle, à là " tunisification » de ces miliciens, qui coupent progressivement leurs liens avec les souverains catalans. Ibid., p. 420.

${ }^{78}$ «Segui ne devint-il pas, dès ce moment, un agent aragonais, s'il ne l'était déjà?»; «C'est [...] Bernat Segui qui est le veritable représentant du roi d’Aragon dans la capitale marocaine». Ch.-Ë. DUFOURCQ, L'Espagne catalane, op. cit., p. 355, 461.

${ }^{79}$ Ibid., p. 488 s.
} 
messagers, chargés de porter les revendications des souverains maghrébins, plus que comme de véritables négociateurs. Ainsi en 1304 Jacques II écrit-il au Mérinide pour lui dire qu'il a bien reçu la lettre portée par Arnau Segui, mais que ce dernier n'a pas mandat du sultan pour négocier, et il demande l'envoi d'un plénipotentiaire pour ce faire ${ }^{80}$. De même en 1312 c'est un musulman que le sultan de Bougie Abû Bakr envoie à la cour du roi de Majorque, avec «pleins pouvoirs pour traiter, s'occuper et conclure la paix et la concorde» ${ }^{81}$. Il y a cependant des exceptions: en 1321 le gouverneur de Bougie envoie un premier émissaire chrétien, Tomas Perez, pour préparer les discussions, puis un plénipotentiaire pour discuter de la paix, mais qui est également chrétien, Joan Botart. Ce dernier ayant fait naufrage, c'est cependant un musulman qui le remplace et effectue la mission, assisté d'un interprète, lui aussi musulman ${ }^{82}$. De même les propositions de paix de 1444 entre les Hafsides et la couronne d'Aragon sont négociées par l'alcayt génois Biasio Cibo comme ambassadeur du roi de Tunis, et qui signe in nomo et per parte de lo dicto Re de Tunisi ${ }^{83}$. Quoi qu'il en soit, c'est au sultan qu'il revient, en dernier ressort, de ratifier les traités ${ }^{84}$ : ainsi l'accord de 1444 doitil être ensuite signé par le sultan ${ }^{85}$ —ce que ce dernier refuse de faire.

En définitive il faudrait plutôt qualifier ces chrétiens envoyés par les sultans maghrébins de messagers, car une fois les échanges préliminaires suffisamment avancés, on préfère envoyer un musulman, qui a toute la confiance du souverain, ou encore mieux finaliser la négociation en terre musulmane.

Il y a donc incontestablement une originalité dans la pratique diplomatique des souverains maghrébins dans leurs relations avec les États chrétiens, aussi bien par rapport à celle que l'on trouve dans l'Europe latine que par rapport à la pratique en vigueur entre souverains musulmans. La faiblesse de la fréquentation par les musulmans maghrébins des États latins oblige à avoir recours à des chrétiens au service des sultans, que ces derniers recrutent dans des populations qui ont l'habitude des contacts entre les deux

\footnotetext{
${ }^{80}$ «no avia mandamiento de vos». Jacques demande l'envoi de «vostros mandaderos». Ch.-E. DufourCQ, Nouveaux documents, loc. cit., p. 302. Il faudrait faire une étude précise du vocabulaire utilisé pour désigner ces différents ambassadeurs, même si celui-ci ne semble pas toujours très fixé. Le termẹ «mandadero» semble en particulier d'un emploi assez systématique, quels que soient les pouvoirs accordés à la personne de l'envoyé.

${ }^{81}$ Plena potestate tractandi et procurandi et faciendi pacem et concordiam. Il se présente comme procurator et nuncius dicti domini regis Bugie. Estanislao de Kostka AGUILÓ, Tractat de pau entre el Rey de Mallorca Don Sanxo y el de Bugia Boyhahia Abubechre, firmat a Mallorca pels seus representants Gregori Sallambé de una part y Mahomat Abdellá ben Acet de l'altra, dia 23 de novembre de 1312, "Bolletí de la Societat Arqueológica Luliana", 15 (1915), p. 218. Il engage alors le sultan cum cartis sigillatis suo sigillo ladabit et aprobabit et ratificabit ea.

${ }^{82}$ Ch.-E. DufourcQ, L'Espagne catalane, op. cit., p. 504-505.

${ }^{83}$ F. CERONe, loc. cit., 1913, p. 32.

${ }^{84}$ À l'inverse le roi d'Aragon donne parfois un parchemin blanc et scellé, et laisse le négociateur libre de conclure un accord. Ch.-E. DUFOURCQ, L'Espagne catalane, op. cit., p. 213, 393.

${ }^{85} \mathrm{Il}$ faudra faire tucti instrumenti carti et scripturi autentici subscripti de manu vestra et sigillati de vestri sigilli. F. CERONE, loc. cit., 1913, p. 32.
} 
mondes, principalement des marchands et des mercenaires. Mais ce qui pourrait apparaître comme une solution risquée pour les souverains musulmans s'avère en réalité assez efficace, dans la mesure où le dîwân reste toujours maître des décisions, ne laissant finalement à ces intermédiaires chrétiens que peu d'autonomie.

Date de reception de l'article: mai 2008.

Date d'acceptation et version finale: juillet 2008. 